\title{
Classification Strategies for Fusing UV/visible Absorbance and Fluorescence Microspectrophotometry Spectra from Textile Fibers
}

\author{
Nathan Fuenffinger, ${ }^{1}$ John V. Goodpaster, ${ }^{2}$ Edward G. Bartick, ${ }^{3}$ and Stephen L. Morgan ${ }^{1}$ \\ 1. University of South Carolina, Department of Chemistry and Biochemistry, Columbia, SC, USA \\ 2. Indiana University Purdue University Indianapolis (IUPUI), Department of Chemistry and Chemical \\ Biology, Forensic and Investigative Sciences Program, Indianapolis, IN, USA \\ 3. George Washington University, Department of Forensic Sciences, The George Washington University, \\ Washington, DC, USA
}

Textile fibers are a frequently encountered form of class evidence in forensic investigations of incidents involving personal contact. Cotton is the most abundant natural source of fibers in the world, and nylon, polyester, and acrylic fibers are three of the most common classes of synthetic fibers likely to be encountered in forensic investigations. As a whole, these fibers are found as trace evidence in more than $80 \%$ of all criminal cases pertaining to textile fibers $[1,2]$.

Initial fiber analysis is often carried out using forms of microscopy. Polarized light microscopy is beneficial towards determining the polymer class (especially for synthetic fibers), and stereomicroscopy enables an examiner to document the physical characteristics of a fiber such as diameter, color, and luster. If the studied fibers, however, are a metameric match, they may not be excluded as originating from the same source. In cases such as these, further analyses can be carried out using optical spectroscopy techniques.

Development of statistical approaches for improving the interpretability of trace evidence analyses has recent emphasis in forensic science. Determining which non-destructive analytical methods will have the highest discrimination power for trace evidence examinations is important so that forensic laboratories conserve time and resources. Knowing which analytical techniques provide complimentary information on the evidence is also useful for ensuring that the data collected is utilized in the optimum manner.

This study compares the discrimination ability of ultraviolet-visible (UV-Vis) micro-spectrophotometry (MSP) and microspectrofluorimetry, two common techniques used by forensic analysts for the analysis of textile fibers. UV-Visible microspectrophotometry is widely used for discriminating fibers based on color [3]. Color is often the most discriminating characteristic of dyed fibers and is the only distinctive feature of many natural fibers such as cottons due to a lack of variation in morphology. To visualize and interpret the vast amount of data that can be collected using one or more spectroscopic techniques, modern statistical methods capable of dealing with high dimensional data are required. Fusion of MSP and MSF data was also evaluated. Low-, intermediate-, and high-level data fusion strategies were employed in discriminations of over 400 dyed textile fiber samples of cotton, acrylic, nylon 6,6, and polyester, resulting in correct classification rates of $97.8 \%, 94.6 \%$, and 93.8\% respectively. Comparatively, classification rates of $89.5 \%, 87.7 \%$ and $87.6 \%$ resulted from quadratic discriminant analysis models built from isolated absorbance measurements, fluorescence measurements with $405 \mathrm{~nm}$ excitation, and fluorescence measurements with $546 \mathrm{~nm}$ excitation, respectively. We find fluorescence to also offer considerable evidentiary value for classification.

Fusion of MSP and MSF data was also evaluated. Low-, intermediate-, and high-level data fusion strategies were employed in discriminations of over 400 dyed textile fiber samples of cotton, acrylic, nylon 6,6 , and polyester, resulting in correct classification rates of $97.8 \%, 94.6 \%$, and $93.8 \%$, respectively. 
Comparatively, classification rates of $89.5 \%, 87.7 \%$ and $87.6 \%$ resulted from quadratic discriminant analysis models built from isolated absorbance measurements, fluorescence measurements with $405 \mathrm{~nm}$ excitation, and fluorescence measurements with $546 \mathrm{~nm}$ excitation, respectively. The results suggest that data fusion is useful for providing additional discriminatory information on textile fibers when compared to single technique data evaluations [4].

\section{References:}

[1] Rendle, D., Wiggins, K., Rev. Progress. Coloration 25 (1995), p. 29.

[2] Palenik, S. Microscopical Examinations of Fibers, In Forensic Examination of Fibres, 2nd ed.; Robertson, J.; Grieve, M.; Taylor and Francis: London, U.K. 1999, pp. 153-177.

[3] Adolf, P.; Dunlop, J. Microspectrophotometry/Colour Measurement. In Forensic Examination of Fibres, 2nd ed.; Robertson, J.; Grieve, M., Eds.; Taylor and Francis: London, U.K. 1999, pp. 251-289.

[4] This project was supported by Award No. 2010-DN-BX-K220 from the National Institute of Justice, Office of Justice Programs, U.S. Department of Justice. The opinions, findings, and conclusions or recommendations expressed in this publication are those of the author(s) and do not necessarily reflect those of the Department of Justice.
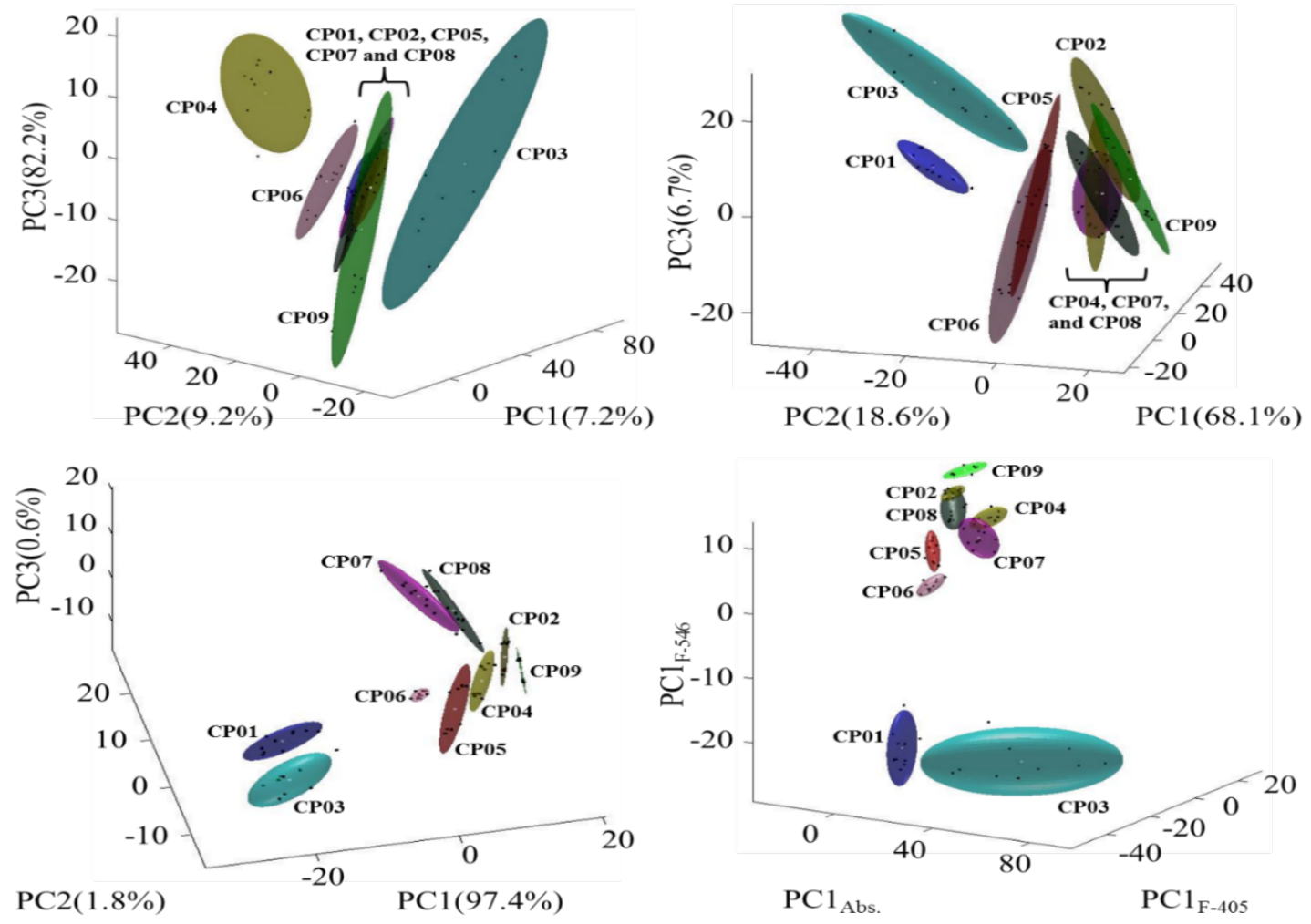

Figure 1. Principal component analysis score plots resulting from absorbance (top left), fluorescence with $405 \mathrm{~nm}$ excitation (top right), fluorescence with $546 \mathrm{~nm}$ excitation (bottom left), and fusion of all three techniques (bottom right) for nine purple cotton fibers. 